\title{
DE NUEVO SOBRE CAJISTAS Y CORRECTORES DE LAS EDICIONES DE LA COMEDIA DE CALISTO Y MELIBEA*
}

\author{
José Luis Canet Vallés \\ Universitat de València \\ Jose.canet@uv.es
}

\section{RESUMEN}

A partir de las comparaciones gráficas entre las tres ediciones conocidas de la Comedia de Calisto y Melibea se intentan analizar las variantes separativas y conjuntivas para reconstruir el árbol genealógico y la transmisión textual de la Celestina de 16 actos. Se pone en duda la primacía de la edición burgalesa (¿de 1499?), y se plantea que todas las versiones siguen al menos una edición impresa de la Comedia (posiblemente la perdida de Salamanca de 1500).

\section{Abstract}

This article aims to analyze the conjunctive and disjunctive variants of the Comedia de Calisto y Melibea, comparing the graphics units of its three known editions, in order to reconstruct the genealogical tree and the textual transmission of the sixteen-act Celestina. It also questions the priority of the Burgos edition (1499?) and it states that all versions follow a lost Comedy (possibly published in Salamanca in 1500).

* Este trabajo se ha realizado en el marco del Proyecto de Investigación FFI2011-25429, concedido por el Ministerio de Economía y Competitividad. 


\section{A Miguel Ángel Pérez Priego, humanista, pero ante todo amigo}

Poco tiempo después de mi edición crítica de la Comedia de Calisto y Melibea ${ }^{1}$ salió una reseña en el Boletín de la Biblioteca Menéndez Pelayo realizada por el profesor Miguel Ángel Pérez Priego en la que, entre muchas cosas, indicaba:

Hay [...] un breve e interesante apartado en que se trata de establecer las características o peculiaridades gráficas de cada una de las tres imprentas y de sus cajistas y correctores. El intento es muy plausible, pero la conclusión es inevitablemente desalentadora: no hay nunca normalización gráfica, pues la actitud de los cajistas oscila entre mantener sus propias peculiaridades y reflejar las grafías específicas del impreso que tiene delante ${ }^{2}$.

En mi edición resumía algunas de las peculiaridades ortográficas de cada imprenta, corrector o cajista, analizando brevemente algunas de las grafías dobles, formas verbales, grupos consonánticos cultos y otras vacilaciones. Intentaré ahora profundizar en dichas peculiaridades por si nos pueden ayudar a una mejor comprensión del estema celestinesco, al menos en sus primeras versiones de la Comedia.

También observé en su momento algunos intentos de regularización de los grafemas y formas verbales en los talleres de los tres impresores, como por ejemplo el verbo vivir, empleado así en la edición burgalesa, frente a $b i v i r^{3}$ en las ediciones de Toledo y Sevilla; la inclusión de la $h$ en el presente del verbo haber: ha y has en el texto sevillano, pero no en las demás formas del verbo (aver, avía, avría), mientras que el de Toledo se decanta por el verbo haver con $h$ en la mayoría de sus formas, menos en la del presente; y el de Burgos prácticamente lo regulariza todo con $h$; la desaparición de la grafía $-f$ inicial (fijo, fija, fallar, fasta, fablar, fecho, fambre, etc.), con un uso regularizado y actual de $h$ en la edición sevillana, pero más medieval con $-f$ en las de Toledo y Burgos. En cuanto a las consonantes dobles, por ejemplo, Burgos suprime todas las doble - ff, que mantiene insistentemente la impresión de Sevilla y también algunas veces la de Toledo (por ejemplo, Sevilla incluye 31 veces la palabra officio/s; Toledo 29; las formas del verbo offrecer, offrescido y sus derivados, aparecen 18 veces en Sevilla, 6 en Toledo; donde se prefiere la simple $-f$, usada en doce ocasiones). Algo similar sucede con la palabra assí, cuyo uso con -ss es la usual en Burgos y Sevilla, mientras que Toledo la simplifica.

La no regularización gráfica en las imprentas (que indicaba Miguel Ángel Pérez Priego), me hizo pensar en la posibilidad de que intervinieran varios cajistas y/o componedores en la estampación de un libro, por lo que me centré en este caso en la Comedia

${ }^{1}$ Comedia de Calisto y Melibea, (2011), ed. crítica de José Luis Canet, Valencia, PUV, col. Parnaseo.

2 Pérez Priego, Miguel Ángel (2012), reseña a «Comedia de Calisto y Melibea, ed. crítica de José Luis Canet, Valencia: Universitat de València, PUV, col. Parnaseo, 2011», BBMP, LXXXVIII, nº 2, pp. 515-517. La cita en pág. 515.

${ }^{3} \mathrm{Si}$ exploramos la mayoría de los textos literarios publicados entre 1495-1505, podemos comprobar que la forma mayoritaria es la de bivir, que prácticamente cuadriplica a la de vivir (búsqueda realizada en CORDE, Corpus Diacrónico del Español, que aunque tenga algunos datos no excesivamente fiables a partir de las transcripciones de ciertos textos incluidos en la base de datos, al menos da un tanto por cien muy elevado de resultados fidedignos). 
de Calisto y Melibea impresa en Toledo, puesto que era la que mayores imperfecciones tenía a la hora de su composición (fallos en la cuenta del original, añadidos de palabras para cuadrar las páginas, etc.). ${ }^{4}$ Para ello dividí el texto de la Comedia en cuadernillos, que vienen marcados con las signaturas $a-k$, pero fraccionando cada pliego en las 4 formas necesarias para su impresión, ya que cada cuadernillo está constituido por dos pliegos conjugados, como se indica a continuación:

\section{Cuaderno a}

Pliego a-1 externo_exterior: folios: $1 \mathrm{r}-2 \mathrm{v}-7 \mathrm{r}-8 \mathrm{v}$

Pliego a-1 externo_interior: folios: $1 \mathrm{v}-2 \mathrm{r}-7 \mathrm{v}-8 \mathrm{r}$

Pliego a-2 interno_exterior, folios: $3 r-4 v-5 r-6 v$

Pliego a-2 interno_interior, folios: $3 v-4 r-5 v-6 r$

Por tanto, cabría la posibilidad de que existieran dos o más operarios para la composición del texto, uno para cada pliego del cuadernillo, o incluso para cada forma del pliego (exterior o interior), ya que se componían independientemente.

En mi intento de comprobar si un oficial regularizaba y otro no, he procedido a búsquedas de palabras y grafías incluidas en cada pliego (consonantes dobles: $-f f,-c c$, $-r r,-l l$, grupos $-s c e$ e imperfecto en $-i e$, ciertas palabras con doble $-s s$, etc.) por si nos pudiera dar más luz sobre el proceso de impresión. ${ }^{5}$ El resultado es el siguiente:

\section{Cuaderno a}

Pliego a-1: Externo_exterior: folios: $1 \mathrm{r}-2 \mathrm{v}-7 \mathrm{r}-8 \mathrm{v}$ (rescela, parescen, resplandesciente, -aparece, -parece).

Pliego a-1: Externo_interior: folios: $1 \mathrm{v}-2 \mathrm{r}-7 \mathrm{v}-8 \mathrm{r}$ (offrezco, offrescimiento, effable, accepto, excellentissimo, acaesce, resplandescen, offrescimiento, merescimiento, -resplandecientes)

Pliego a-2: Interno_exterior, folios: $3 \mathrm{r}-4 \mathrm{v}-5 \mathrm{r}-6 \mathrm{v}$ (officio, ofrescido, tollerar, intollerable, padescer, ofrescido, acontescimiento, desseo, -deseada, deseasse)

Pliego a-2: Interno_interior, folios: $3 \mathrm{v}-4 \mathrm{r}-5 \mathrm{v}-6 \mathrm{r}$ (-sofrir, paresce, guarescer, paresce, -reciba, -obedece, -deseada)

\section{Cuaderno b}

Pliego b-1: Externo_exterior, folios: 9r-10v - 15r - 16v (officio, affan, officios, officio, affeto, -sufras, convalesce, desagradescen, envejesce, paresce, padescido, caresciendo,-deseado,-deseo,-deseo)

Pliego b-1: Externo_interior, folios: $9 \mathrm{v}-10 \mathrm{r}-15 \mathrm{v}-16 \mathrm{r}$ (differencia, offrecido, suffrir, -afirma, occulto, peccado, mescer, paresce, -desear, -deseo, -desean, gozariemos)

Pliego b-2: Interno_exterior, folios: $11 \mathrm{r}-12 \mathrm{v}-13 \mathrm{r}-14 \mathrm{v}$ (affecto, -ofrecia, - sufrir, -sufrio, alleluyas, florescen, crescer, paresce, descender, paresceme, contescimientos, -deseo,-deseado,-deseo)

\footnotetext{
${ }^{4}$ Vid. Moll, Jaime (2005-2006), «Un cuaderno mal contado en la Celestina de Toledo, 1500», Incipit XXV-XXVI, pp. 441-444; y Canet, José Luis (2011), «Introducción» a la Comedia de Calisto y Melibea, ed. cit., pp. 114-129.

5 Aquellas palabras que se acercan a la forma actual en el mismo pliego las incluyo con guión, para hacer resaltar las vacilaciones en una misma forma de impresión compaginada por un único cajista (por ejemplo entre doble -ff, y $-f$ sencilla; doble $-s s$ y $-s$; grupos en - sce y -ece).
} 
Pliego b-2: Interno_interior, folios: $11 \mathrm{v}-12 \mathrm{r}-13 \mathrm{v}-14 \mathrm{r}$ (differencia, difficil, suffrir, peresceria, pesces, acontescido, -deseo)

\section{Cuaderno c}

Pliego c-1: Externo_exterior, folios: 17r - 18v-23r - 24v (officio, officios peccadoras, obedescer, conoscer, rescebir, paresce, paresce, ofresce, -recibiente, -deseando, -deseo, -deseos)

Pliego c-1: Externo_interior, folios: $17 \mathrm{v}-18 \mathrm{r}-23 \mathrm{v}-24 \mathrm{r}$ (effecto, efficaz, effectos, effecto, offender, -ofrece, -ofrescimiento, -ofresce, -ofrecimiento, occupados, -ofrescimiento, empescer, paresce, merescimientos, -parece, -recibe, -recibiente, -acrecentaste, -deseo)

Pliego c-2: Interno_interior, folios: 19v-20r-21v-22r (afficion, officio, officio, officio, querrien, paresce, caresce, conosceras, nascer, amanesciesse, -deseo, aviemos, comiemos, dormiemos, querrien)

Pliego c-2: Interno_exterior, folios: $19 \mathrm{r}-20 \mathrm{v}-21 \mathrm{r}-22 \mathrm{v}$ (effecto, difficile, officio, paresce, paresce, parescer,-deseo,-deseo,-desea)

\section{Cuaderno d}

Pliego d-1: Externo_exterior, folios: $25 \mathrm{r}-26 \mathrm{v}-31 \mathrm{r}-32 \mathrm{v}$ (-ofrecimiento, conoscer, paresce, paresce, -parece, -desee)

Pliego d-1: Externo_interior, folios: $25 \mathrm{v}-26 \mathrm{r}-31 \mathrm{v}-32 \mathrm{r}$ (efficacia, difficil, envegescen, crescer,-carecer, -parecen, -parece, dessean, dessean, dessean, dessean, -desea, -desea, -desear, -deseado)

Pliego d-2: Interno_exterior, folios: $27 \mathrm{r}-28 \mathrm{v}-29 \mathrm{r}-30 \mathrm{v}$ (officio, -sofrir, agradescemos, nascer, merescimiento,-agradecer,-recibe, -empece, -querias)

Pliego d-2: Interno_interior, folios: $27 \mathrm{v}-28 \mathrm{r}-29 \mathrm{v}-30 \mathrm{r}$ (offender, affirmas, officio, -sofrir,-sofrir, occupada, paresce, meresce, crescentar, -parece, -parece, -recibir,-padecido,-empecible)

\section{Cuaderno e}

Pliego e-1: Externo_exterior, folios: $33 \mathrm{r}-34 \mathrm{v}-39 \mathrm{r}-40 \mathrm{v}$ (afficion, desseo, acontescido, merescimiento, -parece)

Pliego e-1: Externo_interior, folios: 33v - 34r - 39v-40r (soffrir, offendo, officio, differencia, afficion, -sofrire, -sofrir, peccador, tollerable, paresce, rescibira, merescimiento, resciben, cresciendo, desseado, -deseo, -deseo, teniedes, vies)

Pliego e-2: Interno_exterior, folios: $35 \mathrm{r}-36 \mathrm{v}-37 \mathrm{r}-38 \mathrm{v}$ (soffrir, differencia, - sufre, querras, excellente, rescebi, meresce, merescimiento, -pareces, desseo, desseos)

Pliego e-2: Interno_interior, folios: 35v - 36r - 37v-38r (officio, effecto, offrecer, -sufras, querras, excellencia, paresce, aborresce, guarescer, obedescieran, paresce, conoscer,-offrecer,-recibio,-empecer,-deseo,-deseo)

\section{Cuaderno f}

Pliego f-1: Externo_exterior, folios: 41r - 42v - 47r - 48v (officios, officio, officio, soffrimiento, escallentar, saliemos, teniemos, deziedes, paresces, aderescen, -recibe)

Pliego f-1: Externo_interior, folios: 41v - 42r - 47v-48r (officio, soffrir, soffrir, -sofrir, rescebido, favorescer, paresce, padescer, favorescer, -guarecer, -empecer, -padecian) 
Pliego f-2: Interno_exterior, folios: $43 \mathrm{r}-44 \mathrm{v}-45 \mathrm{r}-46 \mathrm{v}$ (afficion, difficultad, ofrescele, peccado, amanesce, amanescer, paresce, padesce, paresce, ofrescele, paresce, - reciba)

Pliego f-2: Interno_interior, folios: 43v - 44r - 45v- 46r (differentes, officio, officio, afficion, officio, officio, partie (error por partio), favoresceme, parescen, paresciere, -reciba)

\section{Cuaderno g}

Pliego g-1: Externo_exterior, folios: 49r - 50v - 55r - 56v (efficacia, offrecido, officio, -parece, -parece, -crecen, -deseo,-desear,-desearla)

Pliego g-1: Externo_interior, folios: 49v - 50r - 55v - 56r (-ofrecimiento, querrie, parescer, paresce, obedescen, paresce, paresce, agradescido, rescibo-deseos, - deseo)

Pliego g-2: Interno_exterior, folios: $51 \mathrm{r}-52 \mathrm{v}-53 \mathrm{r}-54 \mathrm{v}$ (officio, afforro, difficultades, officio, differencia, -ofrecian, conoscer, -recibia, -deseo)

Pliego g-2:Interno_interior, folios: $51 \mathrm{v}-52 \mathrm{r}-53 \mathrm{v}$ - 54r (affirmas, paresce, paresce, paresce, permanesce, -parecen, -obedecian,-crece,-descrece)

\section{Cuaderno h}

Pliego h-1: Externo_exterior, folios: $57 \mathrm{r}-58 \mathrm{v}-63 \mathrm{r}-64 \mathrm{v}$ (soffrir, merescer, merescimiento, caresciesen, merescer, -merecer, deseoso)

Pliego h-1:Externo_interior, folios: $57 \mathrm{v}-58 \mathrm{r}-63 \mathrm{v}-64 \mathrm{r}$ (efficacemente, soffrir,-sofrir, -sufre, -sufres, -sufrir, merescedor, descaescimiento, merescimiento)

Pliego h-2: Interno_exterior, folios: $59 \mathrm{r}-60 \mathrm{v}-61 \mathrm{r}-62 \mathrm{v}$ (effecto, officiales, difficile, sofrir,-sofrir, occulta mente, meresce,-parece, -parece, -recibido,-deseo, -desea, -deseo, -deseo, -queria)

Pliego h-2: Interno_interior, folios: 59v-60r-61v-62r (paresce, merescer, -recibiria, -recibas)

\section{Cuaderno i}

Pliego i-1:Externo_exterior, folios: $65 \mathrm{r}-66 \mathrm{v}-71 \mathrm{r}-72 \mathrm{v}$ (peccadora, amanesceria, amanescer, parescera, merescer, merescimiento, -parece, -parece, -deseo, -deseo, -querian)

Pliego i-1: Externo_interior, folios: $65 \mathrm{v}-66 \mathrm{r}-71 \mathrm{v}-72 \mathrm{r}$ (-sofrir, parescen, padescer, paresce, amortesce, conoscer, acaescer, paresce, parescian, -recibiran)

Pliego i-2: Interno_exterior, folios: 67r - 68v - 69r - 70v (officio, officio, official, amanesce, paresce, paresce, paresce, parescia, crescido, acaescieron, amanescio, -crece, -crece, -queria)

Pliego i-2: Interno_interior, folios: $67 \mathrm{v}-68 \mathrm{r}-69 \mathrm{v}-70 \mathrm{r}$ (-ofrescimientos, acaescido, -queria)

\section{Cuaderno k}

Pliego k-1: Externo_exterior, folios: 73r - 74v - 79r - 80v (-sufra, amanescer, paresce, amanesce, contescimiento, merescio)

Pliego k-1:Externo_interior, folios: 73v - 74r - 79v - 80r (-sofrir, occasion, liquescer, caresceys, liquescer, abrie)

Pliego k-2: Interno_exterior, folios: $75 \mathrm{r}-76 \mathrm{v}-77 \mathrm{r}-78 \mathrm{v}$ (offensa, -sufriera, succedieron, entristesce, acrescientan, rescibes) 
Pliego k-2: Interno_interior, folios: 75v - 76r - 77v - 78r (effecto, soffridas, suffren, offrezco, occasion, rescibe, rescibe, parescia, -pareces, -recibiesse, -deseo)

En un principio pensaba que algunos de los cajistas normalizarían ciertas formas verbales o grafías según su tradición o formación en la propia imprenta, pero al parecer las variantes entre - esc/-ec se dan en prácticamente todos los cuadernos y en los distintos pliegos; lo mismo podríamos decir de las dobles consonantes. Creía también que el cuaderno mal compaginado a causa de un fallo en la cuenta del original, ya reseñado por Jaime Moll, ${ }^{6}$ podría darnos más luz sobre un componedor principiante con sus propias reglas ortográficas. Sin embargo no es así, pues en la misma forma (una de las caras de los pliegos del cuaderno f) se dan las dos posibilidades de parescer/parecer/parecia y las de soffrir/sofrir. Todo lo cual me hace pensar que el cajista o cajistas siguen un original impreso, al que se le hizo una nueva cuenta para esta edición, posiblemente con un número menor de páginas, y también algunas correcciones de los grafemas en ciertas palabras, caso por ejemplo de bivir, así, etc., que intentan regular en toda la obra (pero incluso así, algunas veces las incluyen sin modificar siguiendo el texto que tienen delante).

Como bien decía Patrizia Botta sobre estas variantes de mano editorial: «Al lado de los errores, también tenemos un gran número de variantes o de libertades que se toman los tipógrafos, o sea, intervenciones editoriales por razones de mercado o de censura: para vender mejor se elimina el arcaísmo que el público ya no entiende y se le moderniza, se quita la lectio difficilior y se la trivializa o se glosa en una serie de $f a$ ciliores al alcance de todos, a la par que se va nivelando y uniformando la retórica o la sintaxtis más ardua del autor...». ${ }^{7}$ Sin embargo, pienso que algunas veces nos pueden dar pistas sobre la fuente que están utilizando: bien de un original de imprenta, bien de una edición ya impresa anteriormente a la que se sigue casi al pie de la letra.

Veamos unos pocos ejemplos de lo que me interesa demostrar. Como he indicado anteriormente, el uso mayor de la doble - ff ocurre en la edición sevillana, se mantiene titubeante en la toledana y se descarta completamente en la burgalesa:

B: la altitud \& enefable gracia $(5 \mathrm{v})$

C: la altitud y eneffable gracia (8r)

D: la altitud \& ineffable gracia $(7 \mathrm{v})$

B: con ella passan el afan cotidiano (8v)

C: con ella passan el affan cotidiano (10v)

D: con ella passan el affan cotidiano (10r)

Pero en el caso concreto de las palabras differir, differencia/s, differentes (normalmente con doble $-f f$ en las ediciones de Toledo y Sevilla), aparecen dos veces con una sola $-f$ y son coincidentes:

Mas o triste que en esto deferimos (Toledo fol. $4 \mathrm{v}$ )

Mas o triste que en esto diferimos (Sevilla fol. $4 \mathrm{v}$ )

\footnotetext{
${ }^{6}$ Moll, Jaime (2005-2006), «Un cuaderno mal contado en la Celestina de Toledo, 1500», Incipit XXVXXVI, pp. 441-444.

${ }^{7}$ Botta, Patrizia (2009), «Problemas filológicos de un texto impreso», Edad de Oro XXVIII, pp. 29-40; la cita en p. 34.
} 
tanta diferencia ay del fuego (Toledo fol. 6r)

tanta diferencia ay del fuego (Sevilla fol. 5v)

Lo mismo sucede con los imperfectos en -ie, corregidos casi por completo en la edición burgalesa mediante la forma actual en -ia, pero que son coincidentes muchas veces en las ediciones toledana y sevillana (si bien esta última los modifica a partir del folio 54):

$\mathrm{C}:{ }^{8}$ juntas comiemos: juntas dormiemos: juntas auiemos nuestros solazes (21v)

D: juntas comiemos. juntas dormiemos. juntas auiemos nuestros solazes (20v)

B: juntas comiamos. juntas dormiamos. juntas hauiamos nuestros solazes (21v)

C: nunca querrian holgar $[\ldots]$ nunca querrien que amanesciesse (22r)

D: nunca querrian holgar [...] nunca querrien que amanesciesse (20v)

B: nunca querrian folgar $[\ldots]$ nunca querrian que amaneciesse $(22 \mathrm{v})$

C: si saliemos por la calle (41r)

D: si saliemos por la calle (39r)

B: si saliamos por la calle (45v)

En C: entero lo querrie tragar por mas apriessa hazer (50r)

En D: entero lo querrie tragar por mas apriessa hazer (47r)

En B: entero lo queria tragar por mas apriesa hazer (55r)

Si a esto añadimos las similitudes entre las ediciones toledana y sevillana ya puestas de relieve en mi edición de la Comedia, todo ello me hace pensar que ambas siguen un mismo texto. Únicamente unas pocas variantes podríamos considerar como separativas entre $\mathrm{C}$ y D:

C: en su processo nuevas sentencias sentia $(1 \mathrm{v})$

D: en su processo nuevas sentencias tenia (1v)

C: a cada santo lado consintio un ladron (3v)

D: a cada costado consintio un ladron $(3 \mathrm{v})$

B y C: venir mansos a la melezina (63r) y (56v)

D: venir mansos a la melena $(53 \mathrm{v})$

Estas variantes pueden tener su explicación lógica. La primera de ellas, el cambio de la primera grafía entre sentia y tenia es un claro error por alteración o confusión de letras, pues la $s$ larga ( $\int$ ) es interpretada por el cajista por una $t$. Para la segunda: santo lado/costado, no tengo una justificación razonada, a no ser que para el revisor del texto, esta frase le recordara la lanzada en el «costado» de Cristo, o simplemente porque no creía que Jesucristo tuviera «santos lados». Y la última: melezina/melena, es una clara corrección de esta errata por parte del revisor sevillano, pues cualquier supervisor de taller de impresión sabía que melena era una piel que se ponía en la frente al buey para que

${ }^{8}$ Utilizo las letras ya consolidadas para indicar las ediciones del estema celestinesco: B (Burgos), C (Toledo), D (Sevilla). 
no se lastimase con el yugo, por lo que «Traher a la melena [era una frase, que significaba] obligar o precisar a uno a que execute alguna cosa, que no quería hacer» (Dic. Aut.). Lo que me hace pensar que la obra burgalesa (que también incluye la errata melezina) y la toledana siguen un mismo arquetipo común, pues ya las ediciones de la Tragicomedia modifican y proponen la forma correcta de melena.

Por tanto, bajo mi punto de vista, las ediciones de Toledo de 1500 y la de Sevilla de 1501 siguen un mismo ejemplar impreso anteriormente; si bien, como ya indiqué en su momento, la Comedia estampada por Polono, aunque corrige bastantes grafías y formas verbales del texto impreso que tiene delante (probablemente la edición de Salamanca de 1500), no pudo impedir que los cajistas siguieran algunas veces las del texto impreso al que copian línea a línea como fórmula más rápida de confeccionar la forma. Y queda también bastante claro, por lo que hemos analizado hasta el momento, la imposibilidad de que la edición sevillana siga a la de Toledo de Pedro Hagembach. En este caso, mi parecer es coincidente con el de Patrizia Botta, cuando afirma que la edición sevillana de Polono no sigue directamente a la toledana, pues hay una serie de variantes que así lo indican, al no poderse llenar las lagunas por conjeturas ni modificar los errores por divinatio:

C: derrocar bonetes [-]

B y D: derrocar bonetes en mi honor

C: teneys y sacays la triaca de mi llaga

B y D: teneys y tratays la triaca de mi llaga ${ }^{9}$

Sin embargo, no es óbice para que las dos sigan otro texto anterior impreso, puesto que las supresiones y adiciones (en mi honor y muchas más) fueron debidas a errores en la cuenta del original en la imprenta toledana, como puse de manifiesto en mi edición de la Comedia; y en el caso del error de C (sacays) es fácilmente comprensible, pues, como ya indiqué, no hubo corrector de pruebas a pie de prensa en el taller de Pedro Hagembach, verificable mediante los fallos en la cuenta del original y por la multitud de errores tipográficos. ${ }^{10}$ Por otra parte, el operario podía tener en mente la frase «sacar la triaca», con el significado de «sacar el mal de una llaga», ${ }^{11}$ aunque esa no fuera la acepción exacta en el texto celestinesco.

Quizás las variantes separativas más difíciles de armonizar al comparar los tres impresos sean:

B: desea y apetece a ti y a otro menor

C: desea y aparesce a ti y a otro menor

D: desea y cobdicia a ti y a otro menor

${ }_{9}$ Botta, Patrizia, (1994), «Otra vez hacia una edición crítica de La Celestina (II)», Actas del III Congreso de la Asociación Hispánica de Literatura Medieval (Salamanca, 3 al 6 de octubre de 1989). Tomo II, Salamanca, Biblioteca Española del Siglo XV - Departamento de Literatura Española e Hispanoamericana, pp. 953-963. Hay edición digital en Alicante, Biblioteca Virtual Miguel de Cervantes, $2005<\mathrm{http}$ ://www.cervantesvirtual. com/obra/otra-vez-hacia-una-edicin-crtica-de-la-celestina-ii-0/>.

${ }^{10}$ Véase la nota 4.

${ }^{11}$ En el sentido que indica el padre Luis de la Fuente en sus Obras espirituales: «De esta ponzoña de la codizia has de sacar la triaca contra ella...», Parte II, Tratado II, Madrid, Imprenta Antonio Pérez de Soto, 1752, p. 619. 
B: huye lo triste y apetece lo delectable

C: huye lo triste y aparesce lo delectable

D: huye lo triste y cobdicia lo delectable

Muy probablemente, el corrector o revisor sevillano no entendió la palabra aparesce en su propio contexto (creo que formaba parte de la obra que tenía delante, probablemente la de Salamanca de 1500), por lo que la modifica por cobdicia, que sí confiere sentido a la frase (algo similar harán los revisores de la edición burgalesa, si bien piensan que aparesce es una errata por alteración de apetesce»).

¿Qué ocurre con la impresión burgalesa? ¿Secunda el mismo texto, probablemente el de Salamanca, como hacen las otras dos ediciones? A partir de las variantes conjuntivas, las tres proceden de un mismo arquetipo $\mathrm{w}^{16}$, como puse de manifiesto en mi edición de la Comedia, y que repito aquí:

B, C y D: agora Eras y Crato, medicos

B, C y D: O piedad de silencio

B, C y D: Estudia, mientra voy yo de [a] le dezir tu pena

$\mathrm{B}, \mathrm{C}$ y D: todo officio de instrumento [estruendo] forma en el ayre su nombre

B, C y D: O que comedor [encomendador] de huevos assados era su marido

B, C y D: laurel blanco, tortarosa [bistorta rosa] y gramonilla

B, C y D: $\mathrm{Y}$ en tierra esta adorando a la mas antigua y puta tierra [vieja] que fregaron sus espaldas.

B, C y D: hijo de la Clandiana

B, C y D: jamas senti peor habito [ahito] que de hambre

B, C y D: oyr que no aquel Antico [Anfión]

B, C y D: porque la providencia [prudencia] es cosa loable

B, C y D: para tu lengua un freno de sosiego [silencio]

B, C y D: pero para alcançar [alçar] el destierro

B, C y D: Viene Sosias [Sosia] llorando

Además, otras variaciones apuntan a que la estampación de Fadrique Alemán de Basilea y la de Pedro Hagembach tienen delante el mismo texto, constatable a partir de las correcciones hechas en la imprenta de Estanislao Polono, que son las adecuadas:

$\mathrm{B}$ y $\mathrm{C}$ : que veer yo no puede

$\mathrm{D}$ : que veer yo no pude

B y C: Quan brevemente puede

D: Quan brevemente pude

B y C: gorgueras, garrumes, franjas, rodeos

D: gorgueras, garvines, franjas, rodeos

Por otra parte, también habrá coincidencias en algunas grafías dobles en los tres textos (y eso que en el de Burgos son muy escasas), caso de la palabra tollerar y su antónimo:

B: no puede mi paciencia tollerar que (2r)

C: no puede mi paciencia tollerar que $(4 \mathrm{v})$

D: no puede mi paciencia tollerar que $(4 \mathrm{v})$ 
$\mathrm{B}$ : o perpetuo intollerable tormento $(2 \mathrm{r})$

C: o perpetuo intollerable tormento $(4 \mathrm{v})$

D: o perpetuo intollerable tormento $(4 \mathrm{v})$

C: o intollerable pestilencia \& mortal te consuma $(33 \mathrm{v})$

D: o intollerable pestilencia \& mortal te consuma (32r)

B: o intolerable pestilencia \& mortal te consuma (36v)

Veamos ahora algunas diferencias que han servido para establecer la primacía y la cercanía al arquetipo $\mathrm{w}^{16}$ del texto estampado en las prensas de Fadrique Biel de Basilea:

$\mathrm{C}$ y D: sacar aradores a pala de açadon

B: sacar aradores a pala $y$ açadon

C y D: al cordon todo de tratarlo

B: al cordon roto de tratarlo

C y D: con turvino, con tutano

B: con turvino, con tuetano

C y D: o cuerda osadia

B: o cruda osadia

C y D: por el alma de mi padre

B: por el anima de mi padre

C y D: Qual medico jamas pidio tal seguro

B: Qual fisico jamas pidio tal seguro

C y D: quien mucho abarca, poco suele apretar

B: quien mucho abraça poco suele apretar

Muy probablemente, los operarios que hicieron la cuenta y corrección de la obra para su estampación en las prensas burgalesas modificaron algunas palabras a partir de su tradición y formación; rectificaron otras a causa del sentido de la frase; y unas pocas fueron ultracorrecciones desafortunadas. Por ejemplo, por tradición podríamos incluir la variante tuetano. En muchas de las obras de cirugía y medicina salidas de las prensas de Juan de Burgos y posteriormente de Fadrique Biel se utiliza preferentemente la palabra tuetano $^{12}$ frente a la forma clásica manejada en la época medieval: tutano. Algo similar podríamos decir de la preferencia de fisico por medico, incorporada también en la tradición de la Tragicomedia, puesto que según Covarrubias en su Tesoro, «físico» es aquel que sabe la «theórica de la medicina, y médicos, en quanto con la práctica nos curan». Y finalmente, la diferencia alma/anima es algo más compleja de explicar, pero que podría proceder del uso y costumbre en la vida real (rezos y testamentos) y en multitud de

12 Así aparece, por ejemplo, en Epílogo en medicina y cirugía o Compendio de la salud humana de Johannes de Ketham. Tratado de la peste de Vasco de Taranta. Liber physiognomiae de Michael Scotus, Burgos, Juan de Burgos, 15 mayo, 1495; etc. 
impresos de la época: «por el ánima...». ${ }^{13}$ Por tanto, en el contexto podría caber perfectamente esta distinción y se podría explicar esta variación a un corrector culto y avezado en lidiar con tratados médicos y religiosos.

El cambio «cuerda/cruda osadía» puede deberse perfectamente a una interpretación de la fuerza del relato; aunque pienso que entraría en el grupo de las ultracorrecciones desacertadas (no será tomada en cuenta en ninguna de las tradiciones de la obra), puesto que lo que intenta decir Celestina en el fragmento es que siempre tuvo bajo control su atrevimiento y por eso salió indemne (cabe también indicar que la misma frase aparece posteriormente: «Alabo y loo tu buen sofrimiento, tu cuerda osadía [...]», 5 Auto). Igual de desafortunadas son las correcciones «pala de açadon» por «pala $y$ açadon», pues forma parte del refrán: «No se saca arador a pala de açadón», y asimismo la de «quien mucho abraça poco suele apretar», que modifica el proverbio «quien mucho abarca poco suele apretar»; cambios que no seguirán las diferentes tradiciones de la Celestina. La única modificación que tuvo repercusión posterior, porque era de sentido común, es la de todo/roto: «Al cordon roto de tratarlo».

Por lo que hasta ahora hemos analizado, no existe una sola variante de la Comedia de Burgos que presuponga una primacía en el tiempo, y hemos podido verificar que aquellas separativas no tuvieron repercusión alguna en la tradición posterior (bien de la Comedia, bien de la Tragicomedia). Sin embargo, son muchas las coincidencias que obligan a un mismo arquetipo: la inclusión de las mismas erratas y grafías implica que las ediciones de Fadrique Biel y Pedro Hagembach dispongan del mismo texto para sus estampaciones, lo que obligaría a retrasar la fecha de la edición de Burgos al menos hasta 1500 (pues posiblemente seguirían la de Salamanca, que es la que nombra Proaza en la impresión de Jofré de 1514). Pero incluso así, me asaltan montones de dudas sobre esta publicación.

Por lo que hasta ahora he revisado, la edición burgalesa es la que más cuidado tiene en la impresión y la que más normaliza según unos planteamientos claros del operario o del maestro impresor (en este caso Fadrique Biel de Basilea). Por ejemplo, es la única versión en la que casi todas las formas del verbo haber (hauer) aparecen con $h$; en donde no existe ninguna doble $-f f,-m m,-p p$, ni tampoco la doble $-c c$; con poco uso de la doble - $l l$ sin sonido palatal (excepto las escasas veces ya comentadas); la desaparición de la doble $-r r$ a inicios de palabra, etc. Un texo en donde se ha regularizado completamente el verbo vivir (viuir) en todas sus variantes; se han modificado todas las formas verbales $-s c$ en $-s z$ (parezca, padezca, merezca, etc.). Por tanto, es una edición muy cuidada, impresa con esmero, con una buena cuenta del original (impreso probablemente) al que siguen, y con un exhaustivo trabajo del o de los operarios que intervinieron preparando el texto que les serviría de guía en la impresión.

Lo primero que me ha llamado la atención es que en la mayoría de las obras escritas en castellano salidas de las prensas de Fadrique Biel entre 1499 y 1500 mantienen consonantes dobles: $-c c,-f f,-p p$, y a veces $-m m$ (aunque corrija en muchos casos el verbo

\footnotetext{
${ }_{13}$ Véase el diccionario CORDE, en donde aparece dicha expresión al menos en 9 casos en el periodo que va de 1495-1500: «por la voluntad o por el anima o por la consciencia» (Rodrigo Fernández de Santaella, Vocabulario eclesiástico, 1499); «ca peligro seria por el anima del...» (Martín Pérez, Libro de las confesiones, 1500); «por mi anima e por el anima del rector...» (Testamento de Juana del Rojo, 1501), etc.
} 
hauer en la forma usada en la Comedia). Así ocurre en las obras: Antonino de Florencia, Suma de Confesion llamada Defecerunt, 1499 (julio); Confesional del Tostado, 1500; La historia de los nobles cavalleros Oliveros de Castilla y Artús de Algarbe, 1499; etc.

Remedios Prieto y Antonio Sánchez al analizar las abreviaturas en cuatro ediciones tempranas de la Celestina, también llegan a una conclusión parecida: «Lo expuesto permite deducir que la edición de Toledo no pudo colacionar su fuente con la edición de Burgos, siendo, por consiguiente, anterior a ésta, o lo que es lo mismo, la edición de Toledo tuvo que ser la más antigua de todas las existentes y muy probablemente teniendo como original de imprenta un manuscrito», ${ }^{14}$ si bien pienso que esta impresión toledana, por lo que he reseñado de sus grafías y variantes, no pudo tener un manuscrito como fuente y tampoco servir de original de imprenta para las ediciones de Burgos y Sevilla.

Pienso que aquellos que han defendido el año de 1499 para la princeps de la Comedia de Calisto y Melibea lo han hecho porque siguen pensando en la participación del Autor en el desarrollo de la obra (de un primer Auto a 16) y posteriormente actuando en los talleres de Fadrique Biel cuando se imprimiría la Comedia (por lo que no necesitaría de los versos de Proaza como corrector y revelador de la autoría de la obra) y finalmente su transformación en 21 Autos para la Tragicomedia. Si ello fuera así, podríamos pensar que la edición de Fadrique Biel contó con la presencia del Autor, o que al menos éste le hizo llegar un original de imprenta manuscrito para su impresión en letras de molde. Pero entonces, ¿de qué rama común proceden todas las variantes conjuntivas entre las tres ediciones, y también las correcciones y modificaciones del texto burgalés de forma desafortunada? ¿Cuál sería la función del corrector Proaza en las versiones posteriores, si los editores de las ediciones toledana y sevillana ya tenían un texto canónico salido de las manos de su autor? ¿No hubieran seguido todos este texto para las reimpresiones, puesto que era menos costoso copiar línea a línea de un libro impreso que de un original manuscrito? Y sin embargo no fue así.

En este supuesto, la Comedia impresa por Fadrique Biel hubiera salido sin ninguna referencia al Autor y sin la carta del «Autor a un su amigo», los versos acrósticos, y los versos finales del corrector Proaza. Por tanto, los preliminares de las versiones de la Comedia de 1500 y 1501 procederían de la pluma de Alonso de Proaza (junto con los versos finales), quien actuaría como corrector y sería el inventor de todos los textos iniciales firmados por el Autor. Por el contrario, si pensáramos que esa hipotética primera edición de Burgos incluía los preliminares, entonces algún librero desaprensivo a fines del XVIII o principios del XIX extirpó más de medio pliego con una manipulación burda para hacerlo pasar por la princeps de la Celestina.

En este último caso y desde la materialidad del texto, lo normal hubiera sido que si el texto burgalés incluía los preliminares con la carta del «Autor a un su amigo» y El «Autor escusándose de su yerro», exigiría medio pliego (4 páginas que ocupan la intervención del Autor en las versiones toledana y sevillana), y por tanto, como todas las edi-

${ }^{14}$ Agradezco a Remedios Prieto y Antonio Sánchez el poder utilizar este artículo: «Las abreviaturas en cuatro ediciones tempranas de la Celestina: Toledo 1500, Burgos 1499-1502 (?), Zaragoza 1507 y Valencia 1514. Catalogación, cuantificación y consecuencias editoriales», presentado en las XIII Jornadas de Trabajo de la Asociación Española de Bibliografía el 20 de diciembre de 2013 en la Biblioteca Nacional de España, y actualmente en prensa en la revista Celestinesca núm. 38 (2014). 
ciones completas de la Comedia, empezaría el Argumento del primer Auto en el folio A $4 \mathrm{r} ;{ }^{15}$ y si incorporara los versos finales de «Alonso de Proaza, corrector de la impressión al lector», que ocupan dos páginas en las otras ediciones, no podría llevar el escudo final de Fadrique Biel, al estar completo el medio pliego final.

Por tanto, nos confrontamos ante el siguiente dilema: o le quitaron a la Comedia de Burgos sus prólogos y epílogos varios siglos después (lo que implicaría que la edición incorporaba algo más de medio pliego anterior a la signatura A), o bien en su traslado a letras de molde en la imprenta de Fadrique Biel, cuando se incluyeron los grabados, el editor eliminó los paratextos conscientemente para que sus ejemplares no tuvieran un precio excesivo en las librerías. La edición de Toledo tiene 80 hojas sin foliar (signaturas $a^{8}-k^{8}$ ); la de Burgos $a^{8}-1^{8}+m^{4}$, es decir 92 hojas sin foliar, y la de Sevilla $a^{8}-i^{8}+k^{4}, 76$ hojas sin foliar. Así pues, la edición burgalesa cuenta con un pliego y medio más que la de Toledo (el coste de los libros venía dado por el número de pliegos), lo que la hacía más costosa, tanto por la impresión como por el precio del papel. Si un editor quisiera diferenciarse de las ediciones existentes mediante la inclusión de grabados, lo más usual es que hubiera suprimido los preliminares y los versos finales de Proaza, ahorrando un pliego, con lo que la obra sería más cara pero todavía competitiva en el mercado nacional al ser superior por las xilografías. Mi opinión es que la edición de Fadrique Biel fue realizada con esmero, con inclusión de grabados, en un intento de superar las ediciones de la Comedia existentes, pero el coste económico era excesivo si añadía dos pliegos y medio más que la de Toledo (y posiblemente de la edición perdida de Salamanca), por lo que el editor decidió suprimirlos en pro de una mejor competitividad en las librerías castellanas.

Por lo que hemos ido desgranando a lo largo de este artículo, únicamente se podría dar una edición como la de Burgos tiempo después de otras existentes en el mercado, y por tanto, pienso que posterior a la de Toledo, pero muy probablemente también a la de Sevilla. La corrección de las grafías de manera tan meticulosa sólo se puede dar en una imprenta con poco trabajo o por el celo de un editor riguroso que quiere hacerse valer en el mercado incipiente del libro y por tanto, desde mi punto de vista, correspondería a los años 1501-1502, periodo en el que Fadrique Biel de Basilea tiene menor actividad. Además coincidiría con ese intento de normalización grafemática de los textos en su imprenta. Sería interesante hacer comparaciones más exhaustivas, que las que he podido realizar para el presente trabajo, entre los textos en castellano salidos de las prensas de Fadrique durante el periodo 1499-1503 para intentar dilucidar la fecha de impresión de la Comedia (para mí cercana al año de 1502), que si bien salió tarde al mercado editorial cuando ya estaba en gestación la Tragicomedia (por lo que no sirvió para ninguno de los árboles genealógicos de la obra) al menos nos dejó un libro muy bien editado con la inclusión de 16 grabados que le han dado valor añadido.

${ }^{15}$ La Comedia de Burgos se inicia en el fol. A 2r (porque se le ha raspado el número romano i de la signatura Aij para hacerlo coincidir con una posible portada de la obra $\mathrm{Aj}$, cuando todo el mundo conocedor de los textos de la época incunable sabe que las portadas no incluyen dicha señal). 
